Bio - grafía. Escritos sobre la Biología y su Enseñanza. ISSN 2027-1034

Edición Extraordinaria. p.p. 178 - 184

Memorias del IX Encuentro Nacional de Experiencias en Enseñanza de la Biología y la

Educación Ambiental. IV Congreso Nacional de Investigación en Enseñanza de la Biología.

\title{
ESTRATEGIAS DE SENSIBILIZACIÓN AMBIENTAL EN EL ÁMBITO ESCOLAR A TRAVÉS DEL SEGUIMIENTO DE LA GERMINACIÓN Y EST ADÍOS PLANTULARES DE ESPELETIA GRANDIFLORA EN EL P.N.N. SUMAPAZ
}

\section{STRATEGIES OF ENVIRONMENT AL AWARENESS IN THE SCHOOL ENVIRONMENT THROUGH THE MONITORING OF GERMINATION AND PLANTING STAGES OF ESPELETIA GRANDIFLORA IN P.N.N. SUMAPAZ}

\author{
Katherine Lara Figueroa ${ }^{1}$ \\ Jorge Cárdenas Gómez ${ }^{2}$ \\ Luis Alejandro Arias Rodriguez ${ }^{3}$
}

\section{RESUMEN}

Se propusieron estrategias que lograron cambiar la actitud de los estudiantes hacia el cuidado del medio ambiente por medio del grupo "Ecosistemas de alta montaña" y la difusión de su trabajo en la comunidad educativa. Se estableció un plan de acción de educación integral que fomentó la sensibilización ambiental a través del estudio de Espeletia grandiflora, como especie insignia del páramo. Se trabajó en clase sobre la importancia de los servicios ecosistémicos, reforzando lo visto con visitas a los ecosistemas más cercanos y al páramo de Sumapaz, donde se recolectó el material sembrado y se le hizo seguimiento al desarrollo de las plántulas en el invernadero escolar. Se tomaron muestras al azar de 50 individuos senescentes del sector de Chisacá (P.N.N. Sumapaz para disectar sus semillas. Se determinó una tasa de germinación de 5,25\% en las semillas sometidas a hidratación y de $0,34 \%$ in situ bajo condiciones semicontroladas. Se describió la duración de la etapa de plántula hasta el desarrollo de las primeras hojas en presencia de los cotiledones, luego de ser trasplantadas. Se encontró que $E$. grandiflora presenta una germinación estacional con formación de un banco de semillas con potencial regenerativo por su estado de latencia. La reducida tasa de sobrevivencia de las plántulas y la longevidad de sus individuos maduros, la determinan como un ecotipo no pionero. Se comprobó que la participación activa de los estudiantes en esta formación, les permitió analizar y proponer soluciones a los problemas ambientales de su entorno, creando conciencia ambiental.

PALABRAS CLAVES: sensibilización ambiental, germinación, desarrollo plántular, banco de semillas.

\footnotetext{
${ }^{1}$ Magister en Ciencias Ambientales Docente I.E.D.T. Rodrigo de Triana

${ }^{2}$ Magister en Ciencias Ambientales Docente I.E.D. Tom Adams

${ }^{3}$ Msc. Docente Universidad de Bogotá Jorge Tadeo Lozano.1larafigueroak@gmail.com Autor corresponsal.
} 


\title{
Bio - grafía. Escritos sobre la Biología y su Enseñanza. ISSN 2027-1034
}

Edición Extraordinaria. p.p. 178 - 184

\author{
Memorias del IX Encuentro Nacional de Experiencias en Enseñanza de la Biología y la \\ Educación Ambiental. IV Congreso Nacional de Investigación en Enseñanza de la \\ Biología.
}

\begin{abstract}
Strategies were proposed that managed to change the attitude of the students towards the care of the environment through the group "Ecosystems of High Mountain" and the diffusion of their work in the educative community. A comprehensive education action plan was established that promoted environmental awareness through the study of Espeletia grandiflora, as a flagship species of the páramo. The importance of ecosystem services was reinforced through visits to the nearby ecosystems and the Sumapaz moor where the seeded material was collected and the development of seedlings in the school greenhouse was monitored. Fifty senescent individuals from the Chisacá sector (PNN Sumapaz to dissect their seeds) were randomly sampled. A germination rate of $5.25 \%$ was determined in seeds subjected to hydration and $0.34 \%$ in situ under semi-controlled conditions. The duration of the seedling stage was described until the development of the first leaves in the presence of the cotyledons, after being transplanted. It was found that $E$. grandiflora presents a seasonal germination with the formation of a seed bank with regenerative potential due to its state of latency. The reduced survival rate of the seedlings and the longevity of their mature individuals determine it as a non-pioneer ecotype. It was verified that the active participation of the students in this formation allowed them to analyze and propose solutions to environmental problems of their environment, creating environmental awareness.
\end{abstract}

KEYWORDS: environmental sensitization, germination, seedling development, seed bank.

\section{INTRODUCCIÓN}

Los estudiantes del I.E.D. Tom Adams no muestran interés en el cuidado del entorno, desconocen los ecosistemas que les rodean y los beneficios que se obtienen de ellos. Se realiza la pregunta de investigación ¿Qué estrategias deben implementar los docentes del I.E.D. Tom Adams para sensibilizar ambientalmente a los estudiantes? ¿Cómo pueden aplicar el método científico los estudiantes a la solución de problemas ambientales?

Al aplicar las perspectivas y aplicaciones de la educación ambiental de Souvé (1994): en la ambientalista se orienta a resolver problemas ambientales de las comunidades basada en la ecogestión, con el propósito de optimizar la calidad de vida. En la educativa, se centra en el desarrollo personal y colectivoe interacción con el ambiente; y en la pedagógica, se rescata los procesos de enseñanza-aprendizaje que apoya la construcción de un conocimiento analítico y útil a la sociedad. Así que nos planteamos en nuestro hacer pedagógico: ¿Cómo enseñar la educación ambiental a los niños? ¿Hasta dónde se puede permitir la intervención y uso de la naturaleza para que el hombre logre sus propósitos?

Desde esta perspectiva debemos generar en la ciencia y en los seres humanos modificaciones a sus métodos, objetivos y conductas, que permitan encontrar la manera de propiciar en nuestro niños y adultos conciencia de que vivimos en un mundo 
Bio - grafía. Escritos sobre la Biología y su Enseñanza. ISSN 2027-1034

Edición Extraordinaria. p.p. 178 - 184

Memorias del IX Encuentro Nacional de Experiencias en Enseñanza de la Biología y la

Educación Ambiental. IV Congreso Nacional de Investigación en Enseñanza de la Biología.

globalizado pero fragmentado, productivo pero empobrecido, diverso pero intolerante y violento; con riqueza ambiental pero en deterioro (Freire, 1970).

Según Freinet (1996), necesitamos una pedagogía de estrategias, técnicas y recursos, realista y práctica. Las enseñanzas parten de la observación de la vida cotidiana y de la sabiduría campesina. Su pedagogía es renovadora, activa, popular, natural, abierta, cooperativista y se centra en la renovación del ambiente escolar y en las funciones de los maestros, su objetivo es que los niños aprendan haciendo y hagan pensando en nuestra labor docente.

\section{METODOLOGÍA}

Se conformó el grupo de ecología con 35 estudiantes de la básica y media vocacional del I.E.D Tom Adams, localidad de Kennedy en Bogotá, a los cuales se aplicó un test sobre agricultura y ecosistemas. Se creó el centro de interés "Ecosistemas de alta montaña", que por medio de recorridos por los humedales de La Vaca, El Burro, Parque Timiza, los formó en educación ambiental.

Se aplica un formato para tomar las perspectivas antes y después de la salida al Páramo de Chisacá ubicado en la entrada del PNN Sumapaz. A través de una encuesta bajo la técnica de los círculos significativos, los estudiantes plasmaron las personas, actividades, aficiones e instituciones que son importantes para ellos. Los estudiantes aplican los conocimientos adquirido sobre el método científico para analizar y concluir el resultado de sus observaciones. Surgió el interrogante sobre la producción de semillas, la germinación y el crecimiento de nuevas plántulas de E. grandiflora, como especie representativa y vulnerable del páramo.

Durante el trabajo de campo se recolecta el material biológico de este frailejón alrededor de la laguna de Los Tunjos, en las coordenadas $\left(4^{\circ} 17^{\prime} \mathrm{N}\right.$ y $\left.74^{\circ} 12^{\prime} \mathrm{W}\right)$ a una altura de 3.720 m.s.n.m. El muestreo se realiza en la época lluviosa (entre octubre y noviembre de 2014) colectándose capítulos de 50 plantas.

2048 de las semillas disectadas por los estudiantes en el laboratorio, fueron sembradas en el páramo a final de 2014, en bandejas de germinación con tierra cernida del sitio a 0,5 $\mathrm{cm}$ de profundidad, luego cubrieron las bandejas con una malla para evitar la intervención de los animales y las ubicaron bajo un arbusto en campo. Las bandejas se regaron cada ocho días a capacidad de campo. Otras 384 semillas fueron sembradas bajo condiciones de invernadero en el colegio, se mantuvieron hidratadas, expuestas a luz día, con temperaturas entre 10 y $24^{\circ} \mathrm{C}$. Se realizó el seguimiento de las semillas durante seis meses.

Se sembraron a principios de 2015, 972 semillas en bandejas plásticas con papel absorbente bajo condiciones de saturación, separadas por una malla plástica donde en cada celda colocaron cuatro semillas por capítulo, el borde se marcó con el número del individuo y del eje floral que provienen y cubrieron las bandejas con plástico para evitar su 
Bio - grafía. Escritos sobre la Biología y su Enseñanza. ISSN 2027-1034

Edición Extraordinaria. p.p. 178 - 184

Memorias del IX Encuentro Nacional de Experiencias en Enseñanza de la Biología y la

Educación Ambiental. IV Congreso Nacional de Investigación en Enseñanza de la Biología.

deshidratación por evapotranspiración, a temperaturas entre 10 y $20^{\circ} \mathrm{C}$ y expuestas a luz día. El seguimiento se realizó hasta mayo de 2015. Registraron los días de germinación a formación de cotiledones.

A partir de las semillas germinadas en condiciones de saturación, obtuvieron 39 plántulas en estado de cotiledón, que trasplantaron a una bandeja con tierra del páramo bajo condiciones semicontroladas $\left(20^{\circ} \mathrm{C}\right)$, se mantuvo la tierra humedecida a capacidad de campo y expuestas a luz día; la bandeja la cubrieron con un plástico traslúcido para protegerlas de la deshidratación. Se observaron entre febrero y mayo de 2015 (12 semanas) y se calculó la tasa de sobrevivencia.

\section{RESULTADOS}

Según el test diagnóstico, el $90 \%$ no consideró importante cultivar ni conocer sus requerimientos hídricos, el 100\% creyó que la agricultura ecológica puede proteger el medio ambiente y aprendieron algo nuevo al visitar el Páramo de Sumapaz, les gusta trabajar en equipo en la huerta, el $90 \%$ desean continuar con el trabajo en el invernadero y creyeron que el conocimiento de otras materias es indispensable para su mantenimiento; al $100 \%$ le pareció agradable el trabajo en el laboratorio y en el invernadero y les gustaría practicar agricultura urbana en casa.

Luego de las salidas a los ecosistemas y el trabajo de campo, se hizo una reflexión sobre el estado actual de los páramos, a través de un instrumento diagnóstico para obtener ideas para su conservación y conocimiento.

En el laboratorio, los estudiantes observaron que de 972 semillas puestas a germinar en condiciones de saturación, solo el 5,25\% (51 semillas) alcanzaron éste proceso. De las 2.048 semillas, se registró la germinación de siete plántulas en el invernadero hacia junio de 2015 (0,34 \% de germinación), mientras que las semillas bajo condiciones semicontroladas en el páramo no germinaron.

Durante 12 semanas se observaron 36 plántulas con mortalidad del 82,35\%. Desarrollaron sus dos primeras hojas en 14,81 días. A las 12 semanas, el $11,76 \%$ de las plántulas desarrollaron seis hojas, al perder los coltiledones se marchitaron y murieron. La sobrevivencia disminuyó progresivamente, evidenciando un cuello de botella fuerte en la dinámica poblacional de E. grandiflora, que puede estar sujeta a la fragilidad de la pequeña planta y la vulnerabilidad a la deshidratación.

\section{DISCUSION DE RESULTADOS}

Las prácticas ambientales promueven el interés por el saber, el estudiante tiene un papel principal en el proceso de enseñanza que lo promueve a investigar y proponer soluciones ante problemáticas ambientales. El instrumento diagnóstico permitió determinar el contexto en el que los estudiantes perciben el entorno ambiental y generar pautas para su participación activa en el proceso de sensibilización ambiental. Se evidencia avances en 


\title{
Bio - grafía. Escritos sobre la Biología y su Enseñanza. ISSN 2027-1034
}

Edición Extraordinaria. p.p. 178 - 184

\author{
Memorias del IX Encuentro Nacional de Experiencias en Enseñanza de la Biología y la \\ Educación Ambiental. IV Congreso Nacional de Investigación en Enseñanza de la \\ Biología.
}

este proceso, luego de aplicar la prueba diagnóstica por segunda vez, los participantes reconocen su aprendizaje y expresan el deseo de continuar con el proyecto.

Se generaron nuevos aprendizajes con el acercamiento al entorno natural; llegan a analizan la interrelación ciudad- campo, que proporciona servicios ecosistémicos de los que se benefician y que son afectados por la intervención antrópica.

En la investigación, los estudiantes determinan que la humedad y la luz solar son factores importantes en la germinación de E. grandiflora, obteniéndose un mayor porcentaje de germinación bajo condiciones de saturación, comparado con la germinación en tierra. Analizan el efecto del período de latencia de las semillas, con la reducida germinación en el laboratorio $(0,34 \%)$ que favoreció la emergencia de las plántulas por la humedad y disminuyó la mortalidad en la sequía.

Observaron que las semillas enterradas no tenían la activación de la radiación solar y por consiguiente, temperaturas mayores influenciaron la viabilidad de las semillas que no germinaron. Los estudiantes consultaron que en el metabolismo de las semillas, ciertas sustancias inhiben la germinación condicionando a la formación de un banco de semillas viables en el tiempo. Los jóvenes relacionaron el desarrollo alcanzado hasta las primeras hojas en presencia de los cotiledones, con su importancia como tejido de reserva para alcanzar esta etapa. La alta mortalidad en las plántulas en la transición de la época húmeda, presume que puede ser mayor en la temporada seca, puesto que la alta radiación solar y baja humedad relativa, ocasiona una alta transpiración. La pubescencia escasa y su poco grosor, pudo afectar el equilibrio hídrico, produciendo la deshidratación y el rápido marchitamiento en las plántulas.

\section{CONCLUSIONES}

La participación activa de los estudiantes en ésta investigación, les permite analizar y proponer soluciones a los problemas ambientales relacionados con su contexto, creando conciencia ambiental. Al utilizar los elementos del entorno, se promueve la curiosidad, el deseo de aprender así como promover el uso adecuado de los recursos naturales sin comprometer su existencia. La educación ambiental contribuye con el desarrollo integral del estudiante, permitiéndole reconocer y valorar estos ecosistemas y la conservación de los mismos.

El intercambio de experiencias significativas a través de encuentros académicos en las aulas, permite tener un marco de referencia hacia la ecosensibilización. La investigación cualitativa complementaria, permite discernir desde la perspectiva de los fondos de conocimiento e identidad, las diferentes fuentes de sentido e identidad del estudiante, a través de su visión de sí mismo y del mundo.

Se evidenció que se requiere vivenciar estos ambientes para su sensibilización en cuanto a su existencia, importancia y conservación. Mostraron un impacto positivo en los estudiantes, al interactuar con ambientes naturales y participar en buenas prácticas agrícolas, que les permite comprender que la conservación y restauración de los 
Bio - grafía. Escritos sobre la Biología y su Enseñanza. ISSN 2027-1034

Edición Extraordinaria. p.p. 178 - 184

Memorias del IX Encuentro Nacional de Experiencias en Enseñanza de la Biología y la Educación Ambiental. IV Congreso Nacional de Investigación en Enseñanza de la Biología.

ecosistemas se logra con la vinculación entre los diversos actores a través de la educación ambiental Sandoval (2012).

En esta investigación, la germinación de E. grandiflora es dependiente de factores como la luz y la humedad, puesto que hacen que la semilla entre en latencia estacional, la cual se activa con la época de lluvia, manteniendo la viabilidad de la semilla, lo que promueve la formación del banco de semillas a bajas temperaturas, que plántulas en las épocas favorables y disminuye la competencia intraespecífica entre plántulas. Su proceso de dispersión al caer cerca de la planta madre, le confiere protección de los vientos y abrigo ante cambios drásticos de temperatura, que inciden en su sobrevivencia.

Se observa que las hojas delgadas y poco tomentosas en la plántula no la protegen de la deshidratación, su pequeña raíz no le permite absorber suficiente agua ni fijarse al sustrato, lo que afecta su sobrevivencia. La mortalidad incrementa con la pérdida de sus cotiledones y la longevidad de sus individuos maduros, permite determinar la especie como no pionera en una sucesión luego de un disturbio, sino establecerla como parte de un ecotipo del páramo.

\section{BIBLIOGRAFÍA}

Berry, P., \& Calvo, R. 1989. Wind pollination, self-incompatibility, and altitudinal shifts in pollination systems in the high andean genus Espeletia (Asteraceae). American Journal of Botany, 76 (11), Pp. 1602-1614.

Cárdenas, C., Posada V., C., \& Vargas, O. 2002. Banco de semillas germinable de una comunidad vegetal de páramo húmedo sometida a quema y pastoreo (Parque Nacional Natural Chingaza, Colombia). Ecotrópicos, 15(1), 51-60.

Chawla, L. \& Derr, V. 2012. The development of conservation behaviors in childhood and youth. En. S. Clayton. (ed), The Oxford handbook of environmental and conservative psychology. Oxford University Press. Pp. 527-555

Estrada, C., \& Monasterio, M. 1988. Ecología poblacional de una roseta gigante, Espeletia spicata SCH.BIP. (Compositae), del páramo desértico. Ecotrópicos, 1(1), Pp. 25 39. Universidad Nacional de Colombia, Bogotá.

Freinet, Célestin (1996). La escuela moderna francesa: guía práctica para la organización material, técnica y pedagógica de la escuela popular. Madrid.

Freire, P. 1970.La pedagogía del oprimido. Bogotá. Acción cultural para la libertad. Buenos Aires. Tierra Nueva.

González, E. 2001. Otra lectura a la historia de la educación ambiental en América Latina y el Caribe. Desenvolvimiento del Medio Ambiente. (3) Pp.141-158 
Bio - grafía. Escritos sobre la Biología y su Enseñanza. ISSN 2027-1034

Edición Extraordinaria. p.p. 178 - 184

Memorias del IX Encuentro Nacional de Experiencias en Enseñanza de la Biología y la Educación Ambiental. IV Congreso Nacional de Investigación en Enseñanza de la Biología.

Guariguata, M., \& Azocar, A. 1988. Seed bank dynamics and germination ecology in Espeletia timotensis (Compositae), an Andean giant rosette. Biotrópica, 20(1), 5459.

Herrera, J., Alizaga, R., \& Guevara, E. 2006. Germinación y crecimiento de la planta. Fisiología de la producción de cultivos tropicales.

Maya, G. \& María. 2014. Oferta de semillas germinabilidad y micrositios de establecimiento de Espeletia uribeii Cuatrecasas 1978 en el Parque Nacional Natural Chingaza- Cundinamarca. (Doctoral dissertation, Universidad Nacional de Colombia.

Medina I, Paramo P. 2014. Research in environmental education in Latin America: a bibliometric analysis. Revista Colombiana de Educación, (66). Primer semestre de 2014, Bogotá, Colombia.

Mora-Osejo, L. E. y Fals-Borda, O. 2002. La Superación del Eurocentrismo, Bogotá: Academia Colombiana de Ciencias Exactas, Físicas y Naturales.

Moreno González, L. A. 2008. Estrategias de reclutamiento de Espeletia grandiflora Humb. \& Espeletia killipii Cuatrec. en el Parque Nacional Natural Chingaza. Tesis de maestría en Ciencias-Línea ecología. Bogotá, Colombia: Universidad Nacional de Colombia, Facultad de Ciencias.

Morín, E. 2000. Los Siete Saberes Necesarios para la educación del Futuro. Bogotá: Ministerio de Educación Nacional. UNESCO - ICFES.

Ochoa, K. 1994. Banco de semillas de Espeletia grandiflora. Tesis de pregrado, Pontificia Universidad Javeriana, Bogotá, Colombia.

Pérez G., F., \& Pita V., J. M. 2001. Viabilidad, vigor, longevidad y conservación de semillas. E.U.I. Técnica agrícola de Madrid, 16.

Sánchez Z., J., \& Bonilla, M. A. 2006. Evaluación de la reproducción sexual de Espeletia grandiflora VAR. Multiflora en la Reserva Forestal Municipal de Cogua (Cundinamarca, Colombia). Acta Biológica Colombiana, 11(1).

Sandoval, M. 2012 Comportamiento sustentable y educación ambiental: una visión desde las prácticas culturales. Revista Latinoamericana de Psicología, 44(1), Pp. $181-196$

Souvé, L. 1994. "Exploración de la Diversidad de Conceptos y de Prácticas en la Educación Relativa al Ambiente" En: La Dimensión Ambiental en la Escuela. Ministerio de Educación Nacional - FES. Bogotá.

Velandia Q., D. A., \& Fajardo G., A. 2004. Reproducción y adaptación en vivero de algunas especies representativas en las áreas rurales del Distrito Capital de la Región de Sumapaz. Colombia Forestal, 8 (17). 